\title{
Multiple-bar Nuss operation: an individualized treatment scheme for patients with significantly asymmetric pectus excavatum
}

\author{
Xiao Song Ben, Cheng Deng, Dan Tian, Ji Ming Tang, Liang Xie, Xiong Ye, Zi Hao Zhou, Hai Yu Zhou, \\ Dong Kun Zhang, Rui Qing Shi, Gui Bin Qiao, Gang Chen \\ Department of Thoracic Surgery, Guangdong Provincial People's Hospital/Guangdong Academy of Medical Sciences, Guangzhou 510080, China \\ Contributions: (I) Conception and design: XS Ben, C Deng, D Tian, JM Tang, L Xie, G Chen; (II) Administrative support: G Chen; (III) Provision \\ of study materials or patients: All authors; (IV) Collection and assembly of data: All authors; (V) Data analysis and interpretation: All authors; (VI) \\ Manuscript writing: All authors; (VII) Final approval of manuscript: All authors. \\ Correspondence to: Gang Chen. Department of Thoracic Surgery, Guangdong Provincial People's Hospital/Guangdong Academy of Medical Sciences, \\ Guangzhou 510080, China. Email: chenganggz@aliyun.com.
}

Background: Research into multiple-bar Nuss operations for the treatment of wide-range or significantly asymmetric pectus excavatum is rarely reported. This paper aims to explore the curative effects of multiplebar Nuss operations on wide-range or significantly asymmetric pectus excavatum.

Methods: We reviewed the clinical data of 153 patients with pectus excavatum who were treated in our hospital from September 2006 to August 2014. All the patients had wide-range or significantly asymmetric pectus excavatum and underwent multiple-bar Nuss operations performed by the author.

Results: All 153 patients agreed to undergo the operation. The median age was $17 \mathrm{y}(10.2-41 \mathrm{y})$. The median Haller index was 3.98 (3.2-25). One hundred and fifty-one patients accepted treatment with two bars, and 2 cases accepted treatment with three bars. The median operation time was $123 \mathrm{~min}(65-500 \mathrm{~min})$, the median blood loss was $20 \mathrm{~mL}(2-200 \mathrm{~mL})$, and the median postoperative hospital stay was 6 days (3-33 days). The incidence rates of pleural effusion, pneumothorax and hydropneumothorax that required drainage treatment were $0.7 \%(1 / 153), 1.3 \%$ (2/153) and 3.3\% (5/153), respectively. Displacement of a bar occurred in one case, and bar exposure occurred in 7 cases. Therefore, 2 cases had the bars removed early, within 2 years postoperation. One patient with severe depression (Haller index: 8.8) had an unhealed auxiliary incision of the xiphoid process, and although the incision was cured after the early removal of the inferior bar, the deformity recurred. There were no cases of death. Currently, 51.6\% (79/153) of the cases have had the bars removed. The most recent follow-up revealed that patients' median satisfaction score for the surgical correction effect was 9 points (10 points indicated full satisfaction).

Conclusions: For patients with significantly asymmetric and severely deformed pectus excavatum, the multiple-bar Nuss operation not only is safe and effective but can also achieve a better cosmetic appearance. However, we should continue to explore technical improvements.

Keywords: Multiple-bar; Nuss operation; pectus excavatum

Submitted Oct 16, 2018. Accepted for publication Nov 19, 2019.

doi: $10.21037 /$ jtd.2019.12.43

View this article at: http://dx.doi.org/10.21037/jtd.2019.12.43

\section{Introduction}

Minimally invasive pectus excavatum surgery was invented by Donald Nuss and has been used increasingly widely worldwide (1). This operation technique involves placing a steel bar behind the sternum with the concave side of the bar forward and then holding up the depressed sternum by reversing the steel bar such that the convex side faces forward and outward. The steel bar is shaped according to the symmetry and severity of the chest deformity. More than one steel bar can be used in correspondence to the 

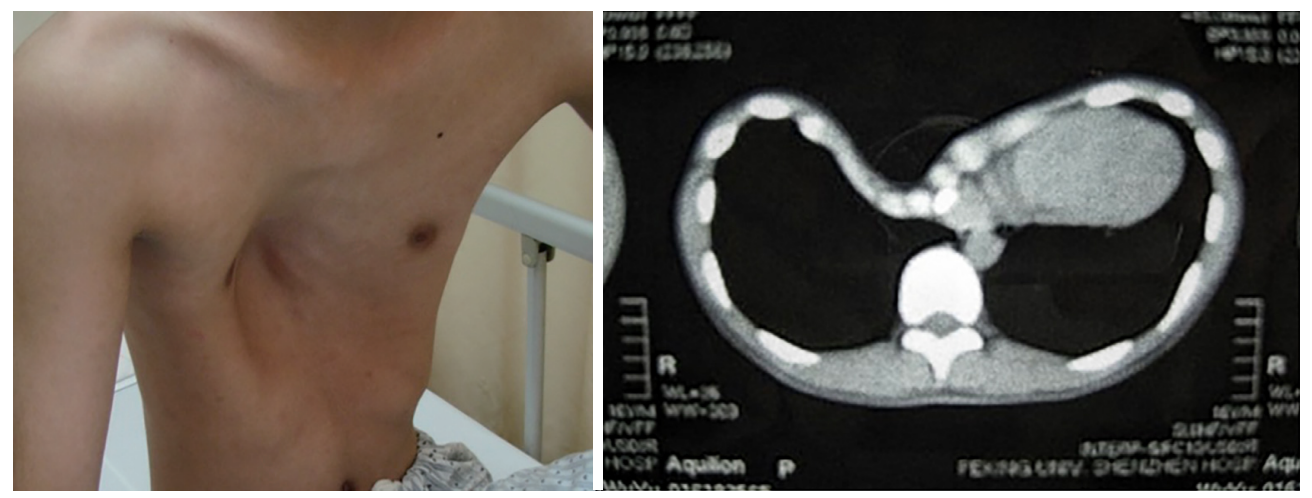

Figure 1 A patient 19-yr-old with marked asymmetries and severe deformities with the deepest portion of the pectus below the spine level.

chest condition of each patient (2). However, the feasibility and effect of the use of multiple steel bars for the treatment of significantly asymmetric pectus excavatum has rarely been reported. Patients with mild, symmetrical pectus excavatum only require one steel bar, but patients with very serious deformities, particularly those with asymmetric pectus excavatum, often require two or more steel bars. This study summarized treatment experiences when two or more steel bars were required.

\section{Methods}

We retrospectively analysed the clinical data of 639 patients with pectus excavatum who underwent thoracic surgery at the Guangdong People's Hospital from September 2006 to August 2014. In all cases, 153 operations requiring multiple steel bars (double steel bars in 151 cases and three steel bars in 2 cases) The median age was 17 years (10.2-41 years). (3). The median Haller index was 3.98 (3.2-25, data missing in 23 cases) (3). Two cases had extremely severe deformities in which the anterior chest wall at the most depressed level nearly reached the rear of the anterior edge of the spine (Figure 1).

The operations were performed after the patients and their parents provided written informed consent. The patients received endotracheal intubation through a single lumen, routine anaesthesia and muscle relaxation. All the patients were given antibiotics $30 \mathrm{~min}$ before the operation. We evaluated the techniques and effects of corrective surgery and summarized the incidence of complications.

\section{Statistical analysis}

All the data were analysed with SPSS 17.0.

\section{Surgical technique}

The patients' thoracic cavities were measured before the operations, and appropriately sized steel bars were selected for shaping on the operation table according to the measured results. The appropriate steel bar was selected such that the bilateral ends were in the area between the bilateral anterior axillary lines and the midaxillary lines based on the shape. Individual shaping technology was adopted according to the specifics of the deformed chest wall. One steel bar was located at the most depressed level of the pectus excavatum, which was usually at the level of the intersection of the point of the fourth intercostal space and the funnel edge, and the other steel bar was placed higher by one or two intercostal levels. In 2 cases of 3 bars, after the insertion of the first bar in the most depressing point and the second bar superior to it, a third bar was placed inferior to the first bar by 2 intercostal space to support the extraordinarily long sternum.

The patient was laid in the supine position with his/her arms extended. A skin incision of approximately $3 \mathrm{~cm}$ was made vertical to the longitudinal axis near each midaxillary line at the most depressed level of the pectus excavatum, and a submuscular tunnel was created along each incision to the anterior chest wall until the intersection of the funnel edge and the pre-arranged intercostal space (Figure 2). One incision was made in each side by the same method at the area one or two intercostal spaces higher. For patients with serious pectus excavatum, an auxiliary incision of approximately $3 \mathrm{~cm}$ long was made in the xiphoid process to uplift the sternum to reduce the difficulty of penetrating the sternum with the perforator and achieving clearance and to reduce the risk of cardiac damage. $\mathrm{CO}_{2}$ was injected via the 

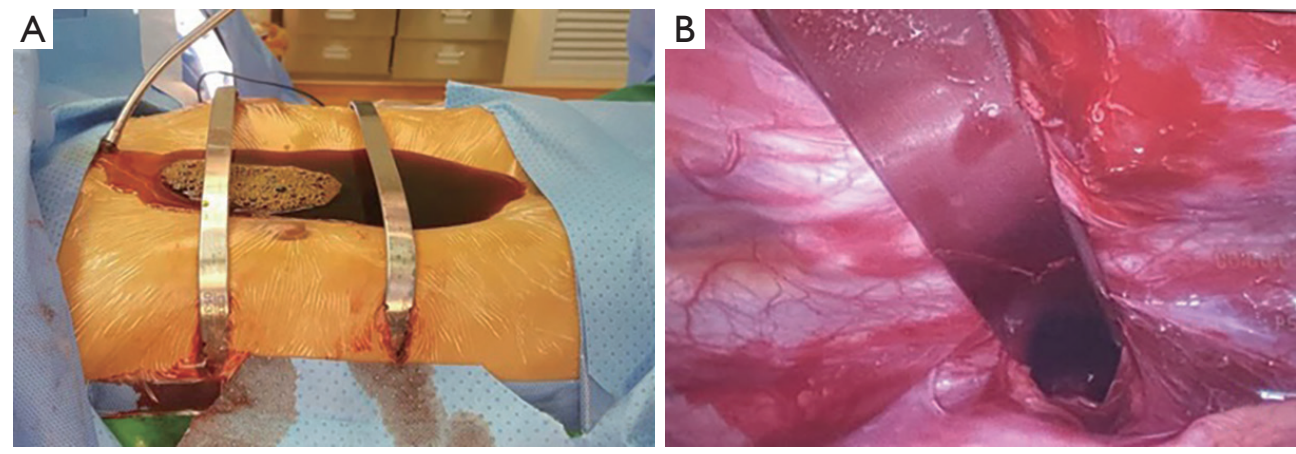

Figure 2 Surgical technique. (A) The bar was bent for the appropriate curves. (B) The incisions into the patients were in line with the deepest point of the depression on the right and left sides.

right low-level incision into the chest cavity to establish an artificial pneumothorax in which to place the thoracoscope, and an S-shaped perforating guider was placed into the tunnel via the right high-level incision under the guidance of the thoracoscope. The terminal end was entered into the chest cavity through the pre-designed intercostal space, carefully moved through the clearance between the anterior mediastinum and sternum while closely adhering to the posterior sternum, and moved out of the chest cavity at the symmetric intercostal space on the left side. Then, the terminal end was entered into the tunnel and moved out of the body via the left incision. The position of the perforator was adjusted to uplift the anterior chest wall, and a yarn belt was then used to penetrate the perforator and the round hole at the terminal end of the steel bar. The yarn belt was tightened, and an end-end connection was created between the perforator and the steel bar. The perforator was extracted slowly through the right side. The extraction steel bar was placed into the chest cavity via the left incision with the convex side of the steel bar backward and moved through the clearance between the sternum and the mediastinum. The perforator position was replaced in the chest, and the terminal end went through from the right incision. A bar turnover device was used to turn over the steel bar by 180 degrees and to hold up the sternum with the convex side of the bar forward. The position of the steel bar was adjusted. A Stabilizer was set at the right end, the steel bar was combined with steel wire or unabsorbable polyester suture (Ethibond), and then the bar was sutured to the lateral chest wall muscle layer. Another steel bar was placed via the low-level incision in the manner way, and the Stabilizer was usually placed in the left side. Gas in the chest cavity was discharged, and the incision was sutured one layer at a time. Routine placement of the chest tube was not needed. Three steel bars were placed in the 2 most serious cases. An auxiliary incision of approximately $4 \mathrm{~cm}$ long was made near the xiphoid process in one case, and part of the rib cartilage was amputated. Postoperative thoracic appearance satisfaction was evaluated by the patients alone or discussing with their parents on a scale on which 10 points indicated full satisfaction. In this group of patients, non-steroidal anti-inflammatory drugs and tramadol were often used for postoperative analgesics. The steel bars were removed 3 years postoperation, and the patients were followed up at 1-month postoperation, 1-year postoperation, and 1 year after the removal of the steel bar.

\section{Results}

One hundred fifty-three patients underwent multiplesteel-bar pectus excavatum surgery from September 2006 to August 2014. One hundred fifty-three patients accepted treatment with two steel bars, and the 2 cases with most serious deformities accepted treatment with three steel bars. One or two Stabilizer were selected according to the stability of the chest wall after the placement of the steel bar to prevent the displacement of the bar. The median operation time was $123 \mathrm{~min}(65-500 \mathrm{~min})$, the median blood loss was $20 \mathrm{~mL}(2-200 \mathrm{~mL})$, and the median postoperative hospital stay was 6 days (3-33 days). Eleven cases (7\%) required catheterization of the chest tube. Postoperative pain was mainly treated with nonsteroidal anti-inflammatory drugs and tramadol. The surgical complications are listed in Table 1 and included single or bilateral pleural effusion in 2 cases (1.3\%), hydropneumothorax in 5 cases $(3.3 \%)$, and pneumothorax in 2 cases $(1.3 \%)$. All of these patients were cured with pleural biopsy or closed drainage of the pleural cavity. Notably, 4 patients exhibited spontaneous 
Table 1 Complications

\begin{tabular}{lc}
\hline Complications & Data \\
\hline Removal of steel bar in advance-exposure of steel bar & $2(1.3 \%)$ \\
Pneumothorax needing puncture or drainage & $2(1.3 \%)$ \\
Pleural effusion needing puncture or drainage & $2(1.3 \%)$ \\
Hydropneumothorax needing puncture or drainage & $5(3.3 \%)$ \\
Fever above $38.5{ }^{\circ} \mathrm{C}$ & $4(2.6 \%)$ \\
Displacement of steel bar & $1(0.7 \%)$ \\
Spontaneous pneumothorax $1-6$ months postoperation & $4(2.6 \%)$ \\
\hline
\end{tabular}
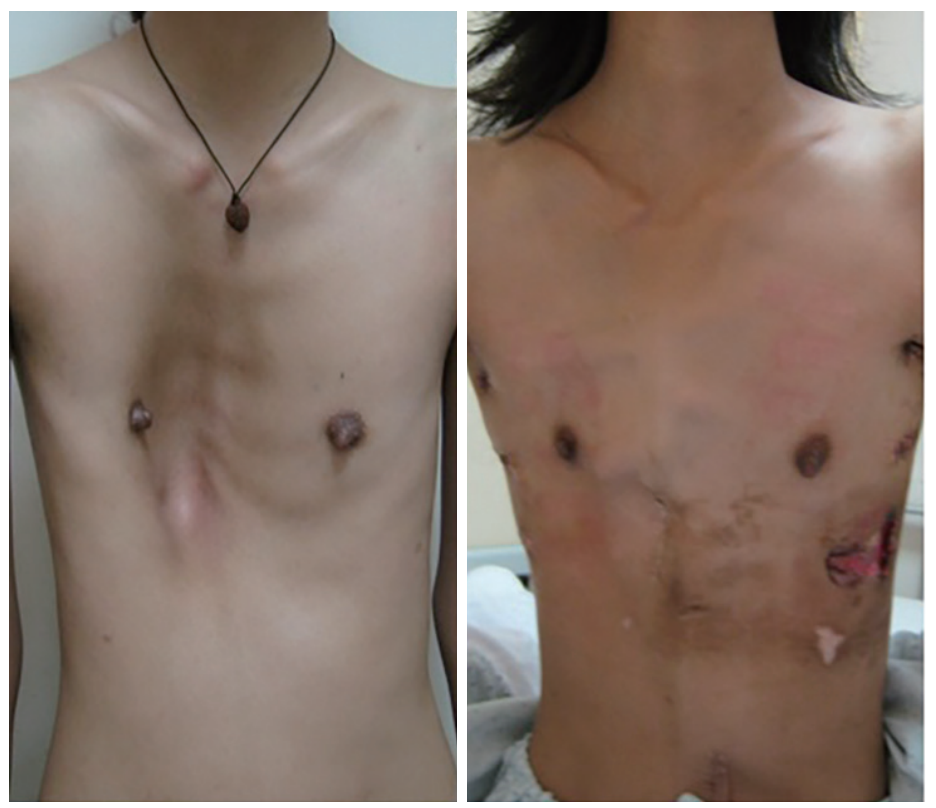

Figure 3 A patient 21-yr-old with 3 bars, experienced incision site infection and healed after bar removal, was grateful for the Nuss procedure for bringing her a marriage and family after the operation (the left panel was collected before the operation, and the right panel was collected after bar removal).

pneumothorax in the postoperative 1-6 months and were cured with closed drainage of the pleural cavity. The steel bars were generally removed after they were retained in the body for 36 months; approximately 51.6\% (79/153) of the patients have had the steel bars removed. Seven cases (4.6\%) experienced the exposure of a steel bar caused by poor healing of the incision. Two of these cases $(1.3 \%)$ underwent early removal of the steel bars within 2 years postoperation, whereas the other 5 cases received routine dressing changes for 2 years and then underwent removal of the steel bars. One case experienced serious displacement of a steel bar; the displaced steel bars were removed 2 months postoperation, and another steel bar was removed after 34 months. The patients with three steel bars appeared to exhibit seriously poor healing of the surgical wound, and dressing changes were the major treatment measure until the removal of the steel bars. Moreover, one patient had a steel bar removed 24 months postoperation and underwent the transfer of a skin flap. The remaining two steel bars were removed 36 months postoperation. This patient is now married and pregnant thanks to the success of the surgery (Figure 3). One patient with a severe depression (Haller index: 8.8) had an unhealed auxiliary incision of the xiphoid process, and the incision was cured after the early 
removal of the inferior steel bar, but the deformity recurred. There were no deaths during the follow-up period. Among all patients, the satisfaction score was 9 points at the last follow-up.

\section{Discussion}

Minimally invasive pectus excavatum surgery has obvious simple and better early effects, has rapidly become prevalent in paediatric surgery and thoracic surgery since its emergence in 1997 and includes the application of one or more steel bars (4-7). However, the related technology and complications of multiple-steel-bar orthodontics in the treatment of patients with significant asymmetry and extremely serious pectus excavatum have rarely been reported. In most cases, one steel bar is sufficient. Occasionally, however, we believe that the use of two steel bars is superior because the chest is unacceptable when the patient moves from the lying position to the standing position. Pectus excavatum surgery should satisfy the patient both in the lying position and in the standing position. The manner in which patients should be selected as suitable cases for multiple-steel-bar treatment and its curative effects has rarely been reported. In our study, we focused on significant asymmetries and serious deformities, including those merged with a flat chest and those with deformed ranges exceeding the length of the sternum length or half of the area of the anterior chest wall, especially when the patients are standing. The more symmetrical and severe the deformity, the larger the space required by the supporting steel bar. Multiple steel bars can fully disperse the pressure to minimize the possibility of displacement of the steel bar to achieve better thoracic cavity shape and relieve postoperative pain (8). We believe that there is now sufficient evidence to prove the adverse effects of serious pectus excavatum on the cardiovascular and respiratory system (9). Sigalet et al. (10) demonstrated that stroke volume, cardiac output, and pulmonary function are all significantly enhanced after pectus excavatum surgery and the removal of the steel bars. Our patients exhibited encouraging results after accepting the treatments with two or more steel bars with positive physical and psychological effects. Moreover, patient happiness was improved.

The advantages of Nuss surgery include minimal invasiveness, a simple operation, a shorter operation time, a stable chest wall shaping and little surgical trauma (11). Although there are various postoperative complications, they are uncommon. The postoperative complications of minimally invasive pectus excavatum surgery (and their estimated incidences) are reported as follows (12-17):

* Displacement of the steel bar requiring another operation $(2.5 \%)$;

* Pneumothorax requiring the placement of a chest tube (3\%);

- Hypercorrection (3\%);

* Epidural catheterization-related complications (4\%);

* Steel bar allergy (2\%);

* Surgical incision infection (1\%);

* Pleural effusion (1\%);

* Thoracic outlet syndrome (0.5\%);

* Pericarditis $(0.5 \%)$;

* Cardiac injuries (0.5\%);

* Sternum injury $(0.5 \%)$;

* Death (0.5\%).

Most complications are associated with the severity of the chest deformity and the lack of operative experience (18). Moreover, with the improvement of surgical techniques and the accumulation of experience, the incidence of complications in Nuss operations will be decreased.

The cases in this study had a low incidence of surgical complications. We found that the patients with complications all had greater and more serious asymmetries and Haller indices, and those with three steel bars were particularly likely to experience longer postoperative hospitalization stays and more complications, primarily because of greater surgical trauma and a higher incidence of operative infection. Pleural effusion, pneumothorax and hydropneumothorax are the most common complications; they can be solved by thoracentesis or drainage. Notably, the steel bar through the retrosternal space impaired the integrity of the anterior mediastinum, which results in the connection of the bilateral chest cavity through this mediastinum defect. In patients with severe pectus excavatum, this defect cannot be completely healed in the short term. Patients with pectus excavatum comorbid by pulmonary bulla and ruptures during this period are prone to serious bilateral pneumothorax, which is difficult to cure by drainage alone; a bullectomy is needed $(19,20)$ All the patients in this group were given a chest CT examination before an operation. Two cases were found to have comorbid pulmonary bulla; both patients were given pulmonary bulla incisions and pectus excavatum surgeries in the same period after we fully communicated with them and obtained their consent. Pneumothorax did not appear after the operations. Four cases of spontaneous pneumothorax, all of which were unilateral, appeared in the 2-6 months 
postoperation. Moreover, the patients were all cured by closed drainage of the chest cavity. Steel bar exposure may be caused by a steel bar allergy or surgical wound infection, which are observed particularly often in thinner patients, and the removal of the steel bar is the solution. Additionally, excessive tension of the steel bar on the skin is one cause of exposure. A shorter steel bar can effectively prevent such exposure and does not affect the corrective effect (21). The reason for this may be that a shorter fixed bar is closer to the anterior chest wall where it has less space to move compared with the lateral chest wall, where longer bars are fixed. Costal clearance for the steel bar to enter in and out is the major location that supports the steel bar. Therefore, the length of the steel bar must only ensure that it does not fall into the chest cavity during development before removal. For adult patients, thoracic development is not considered. We used longer steel bars in the early stages of the Nuss surgeries; these bars could reach the bilateral midaxillary lines, but we then changed and used shorter steel bars that could reach the bilateral anterior axillary line. Additionally, the incision on the side without the Stabilizer was also shorter, at approximately $2 \mathrm{~cm}$. The location of the incision should be placed on the lower edge of the mammary areola, so the postoperative scar is not obvious, and a good cosmetic effect can be obtained. The numbers of cases with long steel bars and short steel bars were similar in number. Seven cases exhibited steel bar exposure, and only 1 of these cases involved the use of a short steel bar. Only 1 case exhibited displacement of the steel bar in this group, and this displacement may have been caused by either an inappropriate postoperative position or a lack of operation experience. Before the maturation of the scar tissue, i.e., for at least 6 weeks after the operation, it is very important to avoid all types of sports (22). We used steel wire to bind the Stabilizer and a steel bar in an 8-character pattern when we began performing the Nuss operations. Although this method has strong fixative effects, the steel wire may occasionally fracture. Moreover, the steel wire should be completely removed during the removal of the steel bar, and an intraoperative chest $\mathrm{X}$-ray film examination is required to avoid wire fracture in vivo, extending the operation time. Later, we used an unabsorbable polyester suture (Ethibond) instead of the steel wire and achieved the same ideal fixative effect while avoiding the risk of wire fracture. We used the method introduced by Park et al. to classify the cases of pectus excavatum and shaped the steel bars according to the actual chest deformities of the patients. For example, asymmetric steel bars were adopted for type 2A, "seagull"- shaped steel bars were adopted for types $2 \mathrm{~B}$ and $2 \mathrm{C}$, and for elderly patients, especially those with osteoporosis, "hump"shaped steel bars were used; all of these bar choices achieved satisfactory corrective results (23).

There were no deaths or cases of thoracic outlet syndrome, pericarditis, cardiac injury or sternum damage. This pattern is attributable to the stability of the multiple steel bars and the application of the thoracoscope, both of which ensured the safety of the operations. During the follow-up period, all patients, including those who had the steel bars removed, were pleased with the effects of the Nuss operation.

\section{Conclusions}

Our research results demonstrated that for complex cases with significant asymmetry and severe deformity, the multiple-steel-bar Nuss operation is safe and effective and can achieve better cosmetic effects. However, we still need a larger sample size and a longer follow-up to fully examine this operation, and it remains necessary to continue to improve the relevant technology.

\section{Acknowledgments}

Funding: This work was supported by Medical Scientific Research Foundation of Guangdong Province, China (A2018139).

\section{Footnote}

Conflicts of Interest: The authors have no conflicts of interest to declare.

Ethical Statement: The authors are accountable for all aspects of the work in ensuring that questions related to the accuracy or integrity of any part of the work are appropriately investigated and resolved. The setting up of retrospective database of $\mathrm{PE}$ was approved by Research Ethics Committee of Guangdong Provincial People's hospital, Guangdong Academy of Medical Sciences. No informed consent or separated ethical review are needed for studies collecting data from this database.

Open Access Statement: This is an Open Access article distributed in accordance with the Creative Commons Attribution-NonCommercial-NoDerivs 4.0 International License (CC BY-NC-ND 4.0), which permits the noncommercial replication and distribution of the article with 
the strict proviso that no changes or edits are made and the original work is properly cited (including links to both the formal publication through the relevant DOI and the license). See: https://creativecommons.org/licenses/by-nc-nd/4.0/.

\section{References}

1. Nuss D, Kelly RE Jr, Croitoru DP, et al. A 10-year review of a minimally invasive technique for the correction of pectus excavatum. J Pediatr Surg 1998;33:545-52.

2. Hebra A. Minimally invasive repair of pectus excavatum. Semin Thorac Cardiovasc Surg 2009;21:76-84.

3. Haller JA Jr, Kramer SS, Lietman SA. Use of CT scans in selection of patients for pectus excavatum surgery: a preliminary report. J Pediatr Surg 1987;22:904-6.

4. Nuss D. Recent experiences with minimally invasive pectus excavatum repair "Nuss procedure". Jpn J Thorac Cardiovasc Surg 2005;53:338-44.

5. Nuss D. Minimally invasive surgical repair of pectus excavatum. Semin Pediatr Surg 2008;17:209-17.

6. Nuss D, Kuhn A, Obermeyer R. Our approach to MIS repair of the pectus excavatum. Contemp Surg 2007;63:444-53.

7. Fang FC, Cheng YL, Lee SC, et al. Clinical experience of Nuss procedure for pectus excavatum in adult female patients. Thorac Cardiovasc Surg 2008;56:283-6.

8. Nagaso T, Miyamoto J, Kokaji K, et al. Double-bar application decreases postoperative pain after the Nuss procedure. J Thorac Cardiovasc Surg 2010;140:39-44, 44.e1-e2.

9. Kelly RE Jr, Cash TF, Shamberger RC, et al. Surgical repair of pectus excavatum markedly improves body image and perceived ability for physical activity: multicenter study. Pediatrics 2008;122:1218-22.

10. Sigalet DL, Montgomery M, Harder J, et al. Long term cardiopulmonary effects of closed repair of pectus excavatum. Pediatr Surg Int 2007;23:493-7.

11. Molik KA, Engum SA, Rescorla FJ, et al. Pectus excavatum repair: experience with standard and minimal invasive techniques. J Pediatr Surg 2001;36:324-8.

Cite this article as: Ben XS, Deng C, Tian D, Tang JM, Xie L, Ye X, Zhou ZH, Zhou HY, Zhang DK, Shi RQ, Qiao GB, Chen G. Multiple-bar Nuss operation: an individualized treatment scheme for patients with significantly asymmetric pectus excavatum. J Thorac Dis 2020;12(3):949-955. doi: 10.21037/jtd.2019.12.43
12. Engum S, Rescorla F, West K, et al. Is the grass greener? Early results of the Nuss procedure. J Pediatr Surg 2000;35:246-51; discussion 257-8.

13. Hebra A, Swoveland B, Egbert M, et al. Outcome analysis of minimally invasive repair of pectus excavatum: review of 251 cases. J Pediatr Surg 2000;35:252-7; discussion 257-8.

14. Marusch F, Gastinger I. Life-threatening complication of the Nuss-procedure for funnel chest. A case report. Zentralbl Chir 2003;128:981-4.

15. Rushing GD, Goretsky MJ, Gustin T, et al. When it is not an infection: metal allergy after the Nuss procedure for repair of pectus excavatum. J Pediatr Surg 2007;42:93-7.

16. Shin S, Goretsky MJ, Kelly RE Jr, et al. Infectious complications after the Nuss repair in a series of 863 patients. J Pediatr Surg 2007;42:87-92.

17. Hebra A, Kelly RE, Ferro MM, et al. Life-threatening complications and mortality of minimally invasive pectus surgery. J Pediatr Surg 2018;53:728-32.

18. Park HJ, Lee SY, Lee CS. Complications associated with the Nuss procedure: analysis of risk factors and suggested measures for prevention of complications. J Pediatr Surg 2004;39:391-5; discussion 391-5.

19. Sakamoto K, Ando K, Noma D. Spontaneous bilateral pneumothorax resulting from iatrogenic buffalo chest after the Nuss procedure. Ann Thorac Surg 2014;98:1463-5.

20. Matsuoka S, Miyazawa M, Kashimoto K, et al. A case of simultaneous bilateral spontaneous pneumothorax after the Nuss procedure. Gen Thorac Cardiovasc Surg 2016;64:347-50.

21. Pilegaard HK. Nuss technique in pectus excavatum: a mono-institutional experience. J Thorac Dis 2015;7:S172-6.

22. Kelly RE, Goretsky MJ, Obermeyer R, et al. Twentyone years of experience with minimally invasive repair of pectus excavatum by the Nuss procedure in 1215 patients. Ann Surg 2010;252:1072-81.

23. Park HJ, Lee SY, Lee CS, et al. The Nuss procedure for pectus excavatum: evolution of techniques and early results on 322 patients. Ann Thorac Surg 2004;77:289-95. 